\title{
Detalles y motivos: Código y decodificación de la medida arquitectónica
}

\author{
Details and motifs: Code and decoding of the architectural meassure
}

$<$ Resumen>

En arquitectura, un detalle plantea siempre un acertijo al momento de advertirse: obliga a descubrir en sí mismo la clave de ingreso a un determinado espacio de referencia mental, el de su significación dimensional.

Esta particularidad recursiva -comprobada aquí en su historia, su evolución y en el versátil estado presente-, lleva a que el detalle sea a veces «motivo de proyecto» y recurso de «escalaje»visual, en la medida que obliga a asumir el «motivo de la escala de ese detalle» allí. Es decir, demanda conocer su razón significativa para la lectura del total.

$<$ Abstract>

In architecture, a detail always presents a puzzle at first glance: to be understood, one needs to discover the clue to access the precise mental space of reference for its dimensional meaning.

This recursive uniqueness -proved here in its history, its evolution and in it's versatile present state- leads to the point where detail is sometimes the project leitmotif and even the means of visual scaling, while at the same time it forces to presume the reason for its actual scale. In other words, its meaningful presence has to be captured to understand the whole.

$<$ PALABRAS CLAVE>

DETALLE ARQUITECTÓNICO / MOTIVO ARQUITECTÓNICO / CLAVES DE ESCALA / «ESCALAJE» / PERCEPCIÓN VISUAL / PERCEPCIÓN DIMENSIONAL

<KEYWORDS>

ARCHITECTURAL DETAIL / ARCHITECTONIC LEITMOTIFS SCALING CLUES / SCALING / VISUAL PERCEPTION I DIMENSIONAL PERCEPTION

\section{Introducción}

Es sabido que ni parte, ni fragmento, ni detalle son lo mismo, aunque en arquitectura tienen cosas en común: en primer lugar, ninguno es abordado en lo que se entiende por «pequeña escala» ya que en rigor ninguno refiere a tamaño ni exige la previa adjetivación dimensional del todo que los contiene para ser comprendido, y ninguno alude a reducción. En segundo lugar, «parte», «fragmento» y «detalle» son siempre capaces de establecer su propia escala si se utilizan como referentes. No obstante, como se demostrará, mientras la «parte» y el «fragmento» en arquitectura siempre pueden revelar su esencia de parcialidad sin recurrir a la escala de su entorno, el detalle exige que la escala de lectura se precise para ser entendido como tal. Porque el detalle arquitectónico, aún teniendo esa misma potencia multiescalar pudiendo asumir cualquier tamaño, tiene características particulares:

* Arquitecta U. de Chile y Doctora en Arquitectura de la Universidad Politécnica de Madrid (2007). Ha sido académica docente e investigadora en México y en Chile. Ha publicado tres libros de la especialidad: "Visualidad y Neguentropía: Equilibrio visual" (1993), con Francisco Brugnoli, "Caleidoscopio de la creatividad" (2001) e "Ingenios de Luz Natural para un Patrimonio Sustentable" (2009) con Cecilia Wolff y Amanda Fuentes, además de numerosos artículos. Ha obtenido numerosas distinciones, siendo galardonada como Mujer Siglo XXI por la Universidad de Chile en 2004. Integra el Senado Universitario entre 1998 hasta 2010.

* Arquitecto U. de Chile 1999, es académica investigadora y docente de la Facultad de Arquitectura y Urbanismo. Es postgraduada de la Universidad de Camerino, Italia, en Historia y Restauración Arquitectónica y actual doctorando de la Universidad Politécnica de Madrid. Ha colaborado con Sofía Letelier desde 1999 en proyectos de investigación y como profesora del Taller de Percepción de la Carrera de Arquitectura. 
- Si bien un detalle es siempre una «parte», ello no es recíproco y no toda parte es detalle. Éste es algo distinto a la partición -lógica o natural- en que dividimos las cosas (pata de una silla, porción de torta), siguiendo la escisión que visualmente sugieren la geometría o las articulaciones de una forma. Porque un «detalle» no es una unidad semática o sema significativo por sí mismo, como sí lo son las partes de una frase o de la arquitectura misma (un «techo» en un edificio o un suburbio respecto a la ciudad), que se segregan naturalmente por su significado propio aunque ligado a su contexto. Pero si las partes no son «detalles», pueden llegar a serlo en algunos casos: cuando algunas se diseñan o disponen con determinada intención llegando a constituir «detalle de tratamiento»-primera acepción disciplinar de «detalle»-; si se proveen «partes» específicas introducidas para reforza las uniones de componentes materiales transformándose en "detalle conector» -segunda acepción-; o si se disponen combinadas en diseños complejos que añaden acentos especialmente insertados como «detalles de complemento»-tercera acepción-, con propósitos puramente preceptuales. Porque los detalles arquitectónicos son ante todo ingenios que dan inflexión a la lectura del todo y que constituyen «articuladores» del interés visual.

- En segundo lugar, el «detalle», por el mismo hecho de carecer de una esencia semática y proveer más bien una regla de lectura, se vuelve atribución «noética» de la sustancia. Es decir, su ser esencial-por ejemplo ser rótula, ser bucle, cantería, u otro- no radica en un significado convenido "por» la arquitectura, sino que descansa en el modo en que se utiliza esa particular solución allí, como decisión localizada. Revelarse como «detalle» depende del rol circunstancial que cumple en el total, y de la norma o código de lectura que su propia presencia establece. Por eso, mientras la «parte» siempre es asociable al todo original, e incluso podemos distinguir a su vez «partes» en un detalle, éstos no pertenecen a la parte que los compone ni al todo: son autónomos como tipo y adquieren su significación sólo cuando se perciben "como detalle». Y un detalle sólo se percibe que lo es cuando se intuye su propósito allí.

- En tercer lugar, el «detalle» difiere también del fragmento porque no tiene su aleatoriedad. El fragmento no responde a una sistémica de partición ni su separación del todo se atuvo a las articulaciones propias de la cosa; y dependiendo de la ruptura causal, resulta tan críptico o comunicativo según las sugerencias que desde sí mismo sea capaz de establecer. Así, mientras la parte aún con significado propio no es nunca autónoma en su significación; y el detalle es autónomo pero como tipo, el «fragmento» mantiene total autonomía significativa. $Y$ es en este aspecto que el fragmento se aleja -más que la parte- de la idea de «detalle»: éste si como tipo puede aplicarse a distintos propósitos y escalas, su materialización no es siempre posible, como sí lo es el fragmento. El detalle existe y significa según su regla y sustancia.

- Un último e importante aspecto distingue a los detalles: por ser códigos o reglas de significación antes que significados, su empleo en una actividad humana como la arquitectura demanda iniciación y una gran capacidad de elocuencia inherente a acto de diseñar. Porque en la arquitectura los detalles fijan y expresan una forma de entender la sustancia material, y manifiestan el modo de vincular y de jerarquizar las partes, comunicativamente: al «detallar» una particular reunión específica de la(s) sustancia(s), cada arquitecto actualiza la capacidad genérica de lenguaje de la especie humana; y con él ejercita el «relatar» cierta forma de hacer, constituyendo el detalle elegido un relato del relato, que es social, ancestral y profundo.

\section{El detalle como ADN: Encriptación de la regla que media entre idea y realidad}

A pesar de la importancia del detalle para la percepción y desciframiento de la arquitectura a lo largo de la historia, el único desarrollo acabado al respecto en la actual disciplina lo aportó Mario Foscari (1984) al inicio del último cuarto del siglo xx. Y resultará útil confrontarlo con las reflexiones que hacía el americano Jonson Ernst (1922) cuando despuntaba el Racionalismo.

Foscari[1] ve al detalle como «vía de conocimiento» de la arquitectura, intrigándole su heurística: esa deriva mental que lleva a concebirlo, a determinarlo y a descifrarlo. Devela un doble sentido en el detalle al señalar que, más allá de mostrar cómo están hechas las cosas, el detalle proporciona una vía consistente para entender «el pensamiento que se ha asociado allí». Insta a mirar en el detalle «más que el logos del techné, la refinada techné del logos»; o sea que más que descubrir en el detalle el conocimiento técnico que lo hizo posible, debe interesarnos cómo determinado conocimiento y manera de pensar se traducen en detalles. Sistematizar su análisis otorgaría a los arquitectos una herramienta para el entendimiento del total «a partir del detalle», supliendo la falta de una aproximación inductiva que vaya, no del todo a la parte-deductiva, como es habitual-, sino de la parte al todo. Por eso, aún si la totalidad arquitectónica es el continente para los detalles, ellos no deben considerarse «elementos subordinados» sino unidades de conocimiento con propia validez. Porque, al estar en ese punto «donde el pensamiento ha resuelto los problemas de la sustancia», logran unificar en sí lo tangible y lo intangible de la arquitectura: unen pensamiento y materia. Son operaciones manifiestas que, en su síntesis, concretan y conectan idea + expresión + posibilidad de ser.

Pero teniendo los detalles un obvio valor estético, hay también en ellos un valor ético. Un ethos los trasciende al concentrar la capacidad individual y social de pensamiento para narrar un relato del hacer. Describen una forma de creer, de pensar y de valorar la sustancia y la técnica, que se vuelve un relatar específico, según sugiere Foscari en CuÉNTAME EL CUENTO, DETALLE. Relatan algo desde cada factor que se conjuga en su resolución y final diseño, siendo fundamental en esto tanto la geometría que envuelven como la disposición que alcanzan en el total: al apreciar una obra, la geometría del detalle es un ancla y una guía visual para el inicio de la ruta mental de apreciación, al proveer los códigos sintácticos presumibles en el resto de la totalidad. Porque no son una cuestión puramente técnica en arquitectura, la construcción geométrica, la decisión numérica y el emplazamiento del detalle «deben considerarse dentro del ámbito filosófico de sus fundamentos y dentro de la teoría de su percepción. Y su geometría, la técnica por la cual el diseñador, el constructor y el usuario transforman, de una mirada, el

[1] Foscari, Mario. 1998. «The 'Tell-the Tail' Detail». En: Theorizing a New Agenda for Architecture. An Anthology of Architectural Theory 1965-1995. Editor. Kate Nesbitt, Princeton Architectural Press. 
signo apropiado en predicción de ocurrencia (en el total) de un cierto fenómeno» ${ }^{[2]}$. Ya que la geometría no establece hechos, sino que provee patrones «en los cuales» podemos o no establecer hechos: provee una estructura para que podamos suponer la existencia de -otros- hechos. De modo que en arquitectura, a nivel de detalles, la geometría y sus principios sólo nos provee una manera de apoyar y de conducir la mirada, en comparaciones que relacionan significativamente los entes articulares percibidos mediante la visión ${ }^{[3]}$

En esta misma línea, otro italiano, Vittorio Gregotti (1983) ${ }^{[4]}$ había anticipado este valor tectónico y ético del detalle que nos permite distinguir la arquitectura de la construcción. por su relación significativa en el conjunto. A su juicio, su valor tectónico -sensualidad física que induce a ver las jerarquías de partes- da siempre claves de escala, al «hacer patente» la relación sustancial, la efectiva y la posible, entre la parte y el todo. Al reconocer en la tectónica el germen de la significación arquitectural, podríamos decir que Gregotti ubica al detalle -consolidado como oficio tectónico- en uno de los extremos del «sentido» que desata la recursividad tautológica en la cual una dimensión -supuesta- nos induce a «escalar» el resto, consecuentemente. Un sentido inductivo del detalle que es opuesto al deductivo que ejerce el territorio o el entorno en la expectativa de dimensión. Por eso la arquitectura -como confirma Juan Ramazziotti (1998)-, sólo manifiesta su presencia como un total sincrónicamente a escala de «lo lejano» (y por sobre los 200 metros): debido a nuestra visión focalizada, no intentamos abarcar comprensiva y visualmente toda la realidad si ella misma no provee, para su entendimiento en profundidad, signos cercanos que atraigan nuestra atención y que conduzcan nuestra mirada hacia otros más allá. En esa actividad de entendimiento espacial dinámico, los detalles que promueven movimiento ocular y la geometría que facilita los desplazamientos visuales - de foco en foco, de accidente en accidente- son «los» vehículos de conocimiento y de «escalaje» en la mente ${ }^{[5]}$
Grabado de Antonio Piranesi, siglo xvIII.

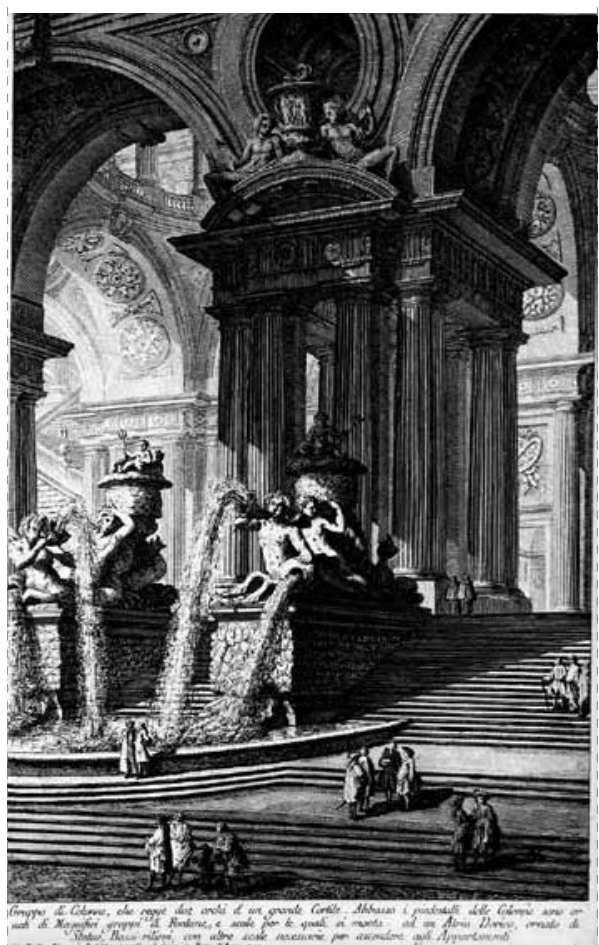

Tal dualidad recursiva del detalle -que es dócil al todo pero que atesora el germen de su decodificación dimensional- se expresa desde el inicio del proceso de proyecto, cuando se define la arquitectura como un sistema de encuentros (físicos) donde ya está el «total». El detalle establece el sistema de relaciones que cuentan el cuento que busca Foscari; un relato cuyo guión consignamos en planos generales y en «detalles» dibujados. Y aún en la arquitectura concebida para provocar tensión mediante elementos extrasistémicos -como fueron las alegorías durante el Barroco, o ciertas trasgresiones a la convención de hoy-, debido a esa misma intención de contravenir lo vigente, son sus detalles -o su ausencia- «la» clave para entender el sistema físico que induce lo visual, en mayor grado cuando éste intenta ser intencionalmente equívoco.
Carlo Scarpa, Museo de Castelvecchio

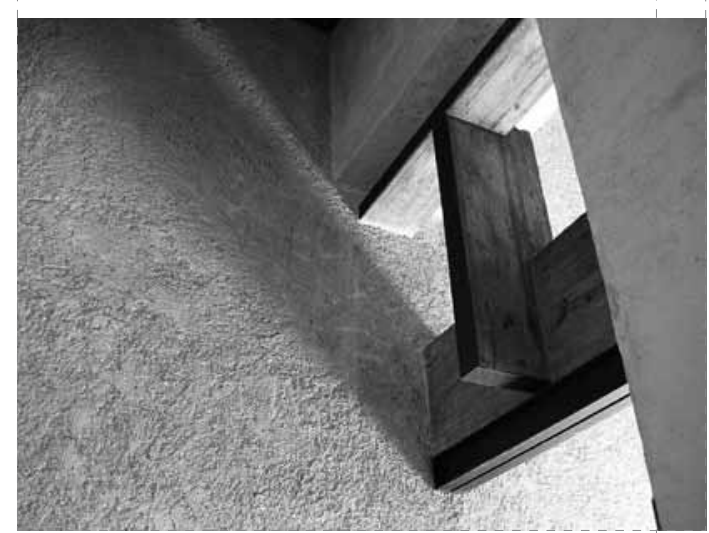

Conceptualización

y evolución del detalle

Hoy el concepto de «detalle» se ha hecho laxo, aunque generalmente se le entiende más como diseño de una unión o inflexión para identificar partes que como el ornamento o agregado plástico que fue en el pasado. La amplitud que abarca hace que se estime como tal incluso aspectos no ideados con ese fin, sea a escala urbana, de edificio o de elemento. Pero a pasar de su indeterminación, «cualquier detalle arquitectónico actúa finalmente como unión», dice Foscari, porque lo percibimos como un mediador. $Y$ en ese carácter, hoy renace del abandono gracias a la compleja mirada posmoderna, opuesta a la desnudez exigida por el racionalismo y antes por Boullée, quien ya en el siglo XVIII instaba a una arquitectura escueta y volumétrica.

Los teóricos franceses del siglo xvIII, al sistematizar en enciclopedias las conquistas constructivas hasta entonces, dieron sin quererlo un nuevo sentido al detalle, aunque manteniéndolo en el ámbito restrictivo de adorno sutil y localizado. A esta limitación habría reaccionado Gianni Baptista Piranesi, difundiendo grabados exuberantes que instaban a aprovechar ese elenco disponible, incidiendo en el posterior eclecticismo. Pero será a partir de las propuestas paralelas

[2] Nota: Foscari se inspira en las enseñanzas de J. Labatut, quien yo también tuve la suerte de conocer, por entonces -1968- en Rice University, EE.UU.: Como dice el profesor de Princeton, Jean Labatut: «Cualquiera sean los espacios, áreas o dimensiones comprometidas en una obra, es el estudio preciso de la buena ejecución de los detalles lo que confirma la buena arquitectura. Los detalles cuentan el cuento».

[3] La importancia de favorecer las conexiones mentales con movimientos oculares reales, y la incidencia de la geometría en tal fenómeno de percepción individual y de la escala, ya las había explicado Hermann von Helmholzt en su MANUAL DE ÓPTICA FISIOLÓGICA (1856), según recoge Foscari textualmente: «El ojo representa un instrumento visual con un muy amplio campo de visión, pero sólo una muy pequeña, confinada y estrecha parte de ese campo produce imágenes claras. La parte más focalizada del campo total se presenta claramente delineada mientras hacia los bordes está meramente esbozado, y crecientemente tosco mientras más nos alejamos del principal objeto de atención. Gracias al movimiento del ojo, no obstante, es posible examinar detenidamente cada punto del campo visual sucesivamente». Foscari, Mario. "The 'Tell-the Tail' Detail» En: Theorizing... Op cit, p. 513.

Gregotti, Vittorio. «The Exercise of Detailing». En: Theorizing... Op cit, pp. 494-498. (Originalmente en Casabella № 492, junio 1983).

[5] Ramazziotti, Juan. 1998. «Huellas Espaciales de la dimensión humana. Hacia un Modelo Integral de las escalas dimensionales del habitar». Tesis Doctoral, ETSAM, UPM. 
y contestatarias de Etienne Louis Boullée y de los arquitectos de la «arquitectura parlante», que el detalle propiamente arquitectónico adquiere valor de código: promovían utilizar sólo aquel detalle que se lograba con elementos de la arquitectura misma ${ }^{[6]}$, desechando los «agregados plásticos» o alegóricos y los ornamentos que se sobreponían a la arquitectura. Tal pureza sintáctica demandó generar durante el Beaux-Arts, un instrumento descriptivo para el detalle, la analítica gráfica, a fin de entender la coherencia del tejido tectónico total. Recién el detalle comenzaba a ser reconocido en la disciplina proyectual como gen de coherencia.

Durante el período preindustrial, el detalle se vuelve ese asunto económico y fáctico, que muchas veces se resuelve por especialistas lejos del arquitecto. Según Foscari, con ello volvía a pensarse reductivamente como «delineación al tamaño 1:1 de cualquier porción de un diseño arquitectónico», dócil a la definición del AmERICAN GLossary of BuILDING, no obstante que el glosario francés de la época ampliaba su comprensión a toda «especificación y descripción del trabajo a ser realizado en la ejecución», aceptando complementarlo con medios verbales o gráficos. Es recién con el movimiento Arts and Crafts en plena época industrial, que el detalle es visto en su doble faz de creación y de trabajo, por la parte del arquitecto y del trabajador ${ }^{[7]}$. Y es hacia 1931, con el pronunciamiento ético y estético de Adolf Loos en Ornamento y delito (1908) -influido por Sullivan y la Escuela de Chicago-, que se consolida la distinción excluyente entre detalle y ornamento, al rechazar enérgicamente éste último si supone desperdicio de trabajo y de material. Según Kenneth Frampton, aunque Loos salvaba del repudio «aquel ornamento espontáneo y artesanal, por ser expresión vernácula», no fue comprendido por los «puristas» del Movimiento Racionalista, que adoptaron de sus postulados sólo el rechazo a la ornamentación, vaciando con ello de contenido la idea misma de detalle ${ }^{[8]}$.

\section{¿Debe el detalle adecuarse a la escala del total, o éste determina la escala del detalle?}

Ya el Renacimiento aplicaba convenciones la correcta disposición de detalles para una adecuada lectura del total. Gianni B. Alberti lo explicitó en De Re Aedificatoria, al concebir la arquitectura como el «arte de lo adecuado», según se infiere de su definición de Belleza: «es la concurrencia (o confabulación) de todos los detalles en la unidad a la que pertenecen ${ }^{[9]}$. Belleza surgida de la habilidad para reunir partes según una norma, de modo que nada pueda ser añadido, restado o alterado sin estropearla, contribuyendo los detalles a su perfección; y donde toda "unión tratada» revela el encuentro entre la capacidad mental de construir y la actual construcción.

Alberti entendía la Belleza como proceso de significación progresiva; de modo que lograr la concurrencia armonizada de todas las partes y detalles era su manera de alcanzarla. Con tres condiciones:

- El Número: dado que siempre hay variados elementos en una obra, es necesario determinar la correlación numérica entre ellos. Si bien para Alberti se trataba de correlaciones cosmogónicas o basadas en el cuerpo humano, hoy la importancia del número se repone avalada desde la ciencia: por la psicología de la percepción, por ejemplo, sabemos que los números pares y los impares -hasta la cantidad de 7- funcionan muy distinto en el anclaje de la atención, en la fijación de focos de lectura, en la escisión del todo, etc., incidiendo fuertemente al captar la naturaleza de una entidad.

- La Inducción Dimensional: en Alberti, los límites de los cuerpos, elementos y detalles instalan automáticamente un sistema de proporciones analógicas que, al leerlas, asumimos como relaciones de medida para todo lo demás. Si ello era válido para el sistema canónico y autorreferido de estilos proporcionales, lo es también hoy en un sistema escalar fenomenológico de «constancias» visuales - a partir de
[6] Boullée, Etienne-Louis. Arquitectura. Ensayo sobre el Arte. Ed. Gustavo Gili, Col. Punto y Línea, Barcelona, 1985. 77] Foscari, Mario. "The 'Tell-the Tail' Detail». Op cit, p. 509

[8] Frampton, Kenneth. Historia Crítica de la Arquitectura Moderna. Ed. Gustavo Gili, 2002, p. 93.

[9] Van Eck, Carolina. «Architecture, Lenguage and Rethoric in Alberti's De Re Aedificatoria». En. Architecture and Language. Constructing Identity in European Architecture. c.1000-1650. Klark, G / Crossley, P. (editores), Cambridge Univ. Press, London, 2000.
Merleau Ponti-, que considera además el efecto de los bordes y del entorno.

- La Colocación: para Alberti, se debía controlar la «composición» mediante la ubicación funcional de los detalles. Función no sólo práctica o tecnológica para él, sino estética y «de memoria». Según la necesidad práctica, visual y significativa, la pertinencia de la ubicación de un detalle resulta aún hoy importante para la expectativa de percepción de las dimensiones y del todo que prevé el proyectista.

El número de partes y detalles -como advertía también Boullée- es capaz de modificar la escala percibida cuando su cantidad es considerable, o mínima o única. Grandes cantidades de detalles en espacios reducidos acentúan la pequeñez, al saturar la visión sin expectativa de descanso, intensificando la decodificación perceptiva. Por otra parte, elementos excesivamente distanciados no inducen fáciles relaciones entre sí, percibiéndose aislados sin pertenencia al conjunto si falta tensión o proximidad para inferir una medida. De modo que la colocación relativa de detalles en el todo promoverá o evitará asociaciones centrípetas o centrífugas que permiten explorar la totalidad interna y su contextualización «en « una escala determinada. Aún hoy, la decisión ex ante de la escala deseada para el total impulsa a decidir asuntos tan primarios como el número, la dimensión y la colocación de detalles, requerimientos albertianos de Belleza. Porque si bien se cuestiona la vigencia de tal concepto, la mirada de Albert orientada a lograr una cualidad del total, por su universalidad y abstracción sigue siendo válida.

\section{Detalles versus «Motivos» en la percepción de escala}

Muchas veces «motivo» y detalle tienden a confundirse; aunque el detalle generalmente nos ubica en el vínculo tectónico u organizativo de la sustancia, mientras del «motivo» esperamos una elocuente singularidad que provoque el reconocimiento del objeto. Y si bien un detalle puede constituirse en "motivo» de carácter, no todos los «motivos» radican en detalles. Pero, a pesar de esta falta de reciprocidad, ambos actúan de forma similar en la comprensión espacial:

- Ambos promueven una articulación interna (quiebre de continuidad) del objeto. 
- Ambos, para ser entendidos, promueven la lectura total del objeto y la lectura de su escala.

- Ambos establecen a su vez su propia escala, desde la cual se juzga la del total.

Sin embargo, la escala de los «motivos» no es la misma que la escala de los detalles. Ello es evidente en los diversos niveles de tratamiento que se les da a los «motivos» en edificios representativos, desplegados gradualmente desde la fachada a zonas intermedias e

interiores. Un pórtico, un remate del volumen, o ductos de circulación expresados como «espacios servidores», constituyen «motivos» de arquitectura que se emplean aún como recursos estéticos, a pesar de las diatribas de racionalismo y de las rebeldías posmodernas. E incluso los mantos y «pieles» más continuos de la arquitectura del cambio de milenio no están carentes de "motivos». Para ellos sigue siendo válido lo que hace casi un siglo observaba Jonson Ernst: «dos factores principales de escala demandan la atención del diseñador:

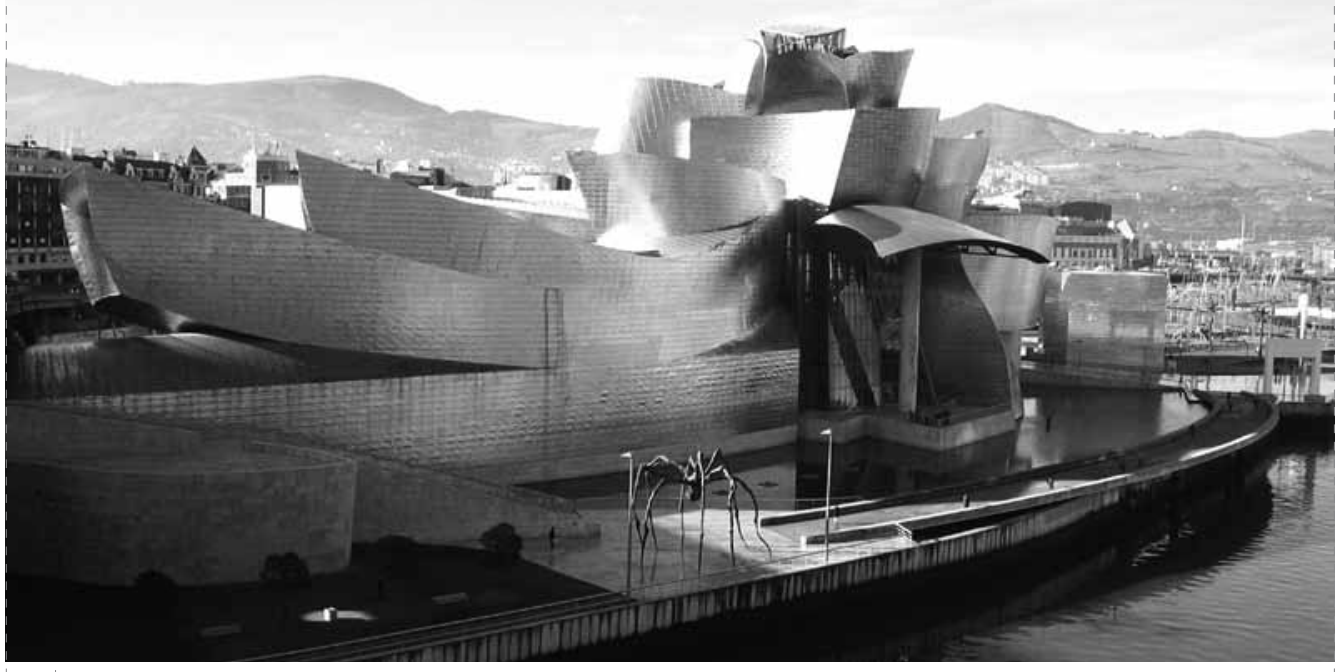

Museo de Bilbao, F. Ghery

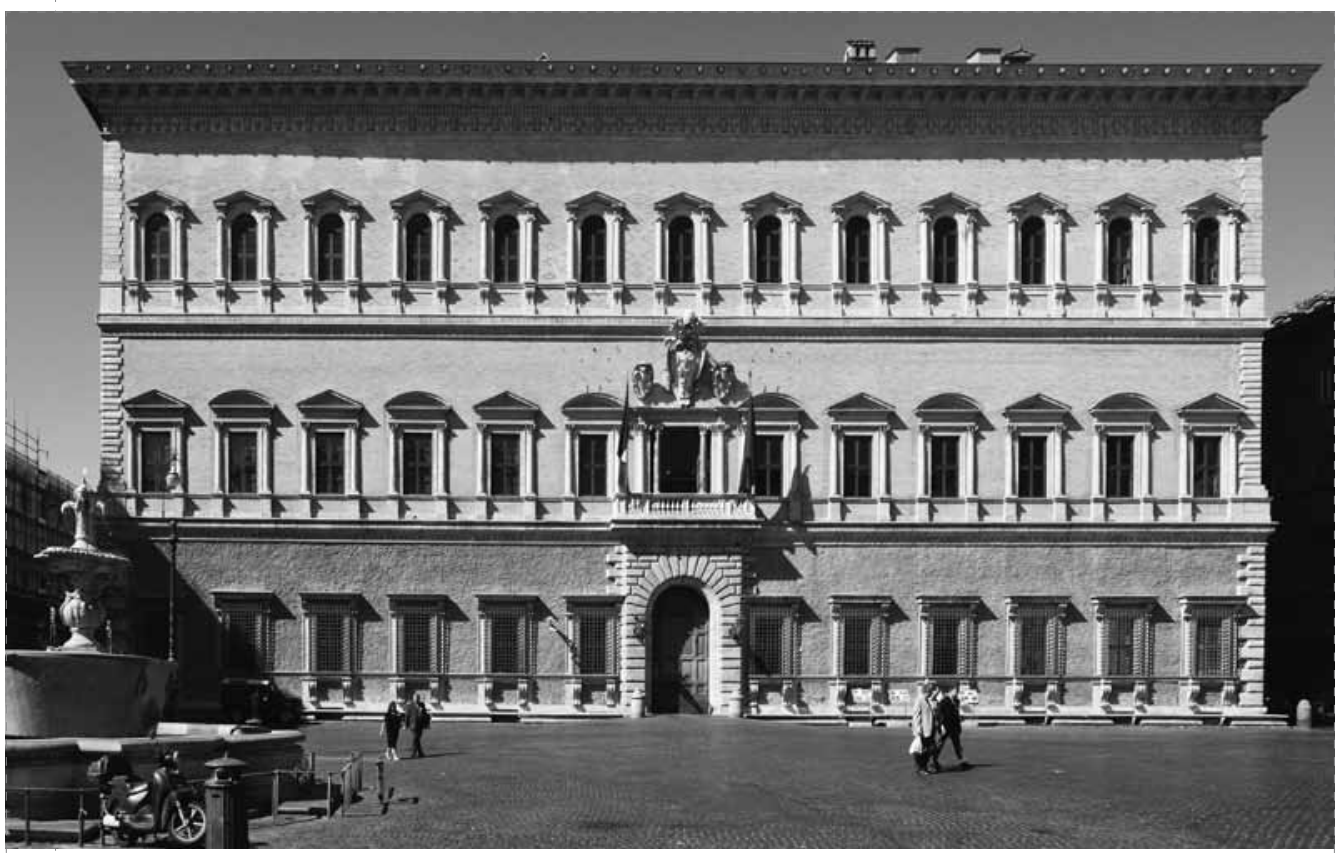

Palacio Farnese, en Roma (fotografía: Myrabella, Archivo de wikimedia commons. Se autoriza la copia, distribución y/o modificación de este documento bajo los términos de la Licencia de documentación (ibre GNU).

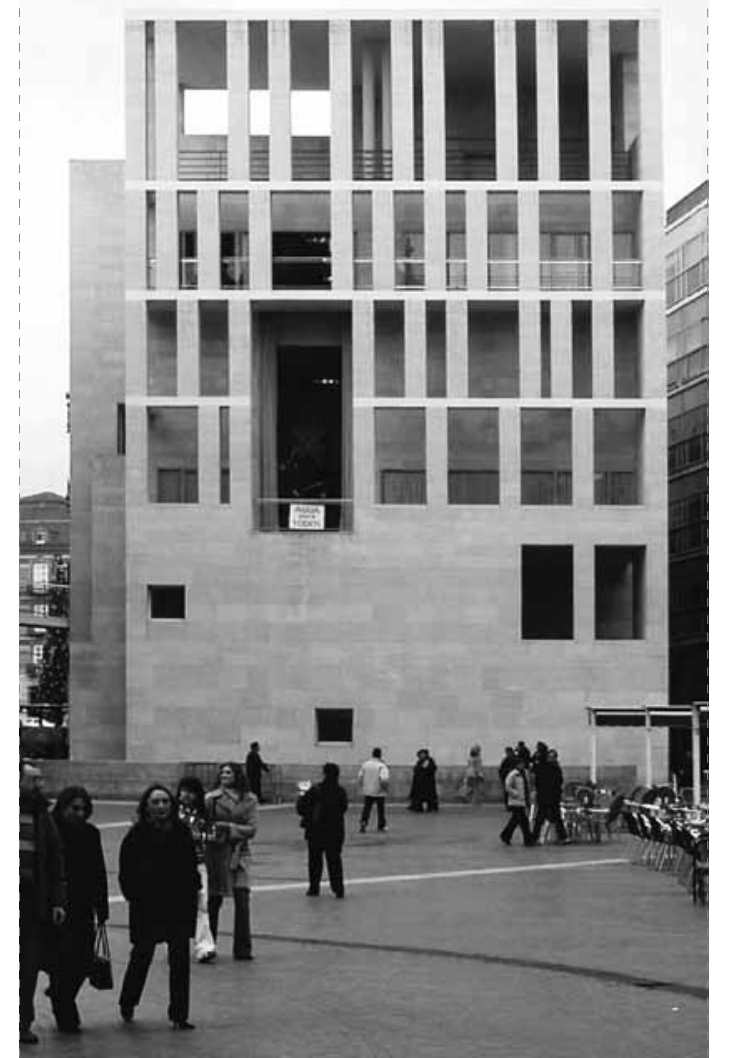

Ayuntamiento de Murcia de Rafael Moneo (fotografía: JCRA. Archivo de wikimedia commons. Se autoriza la copia, distribución y/o modificación de este documento bajo los términos de la Licencia de documentación libre GNUl . 
Parlamento de Escocia. Eric Miralles y Benedetta Tagliabuhe Plafón de la Sala (fuente: Www.arkinetia.com)

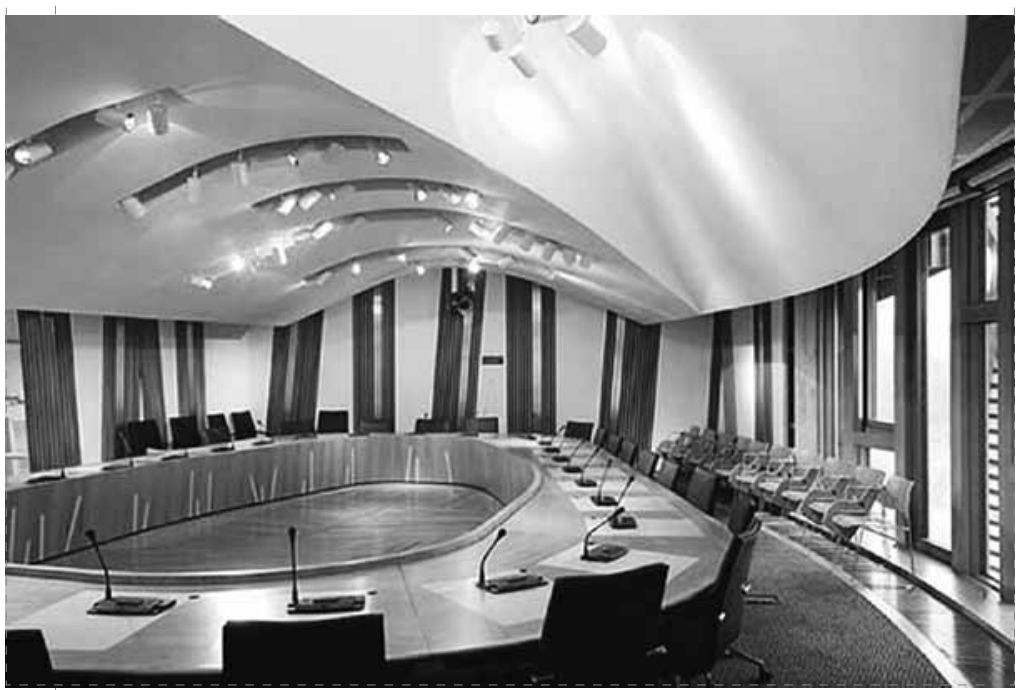

Fachada (fuente: www.arkinetia.com)

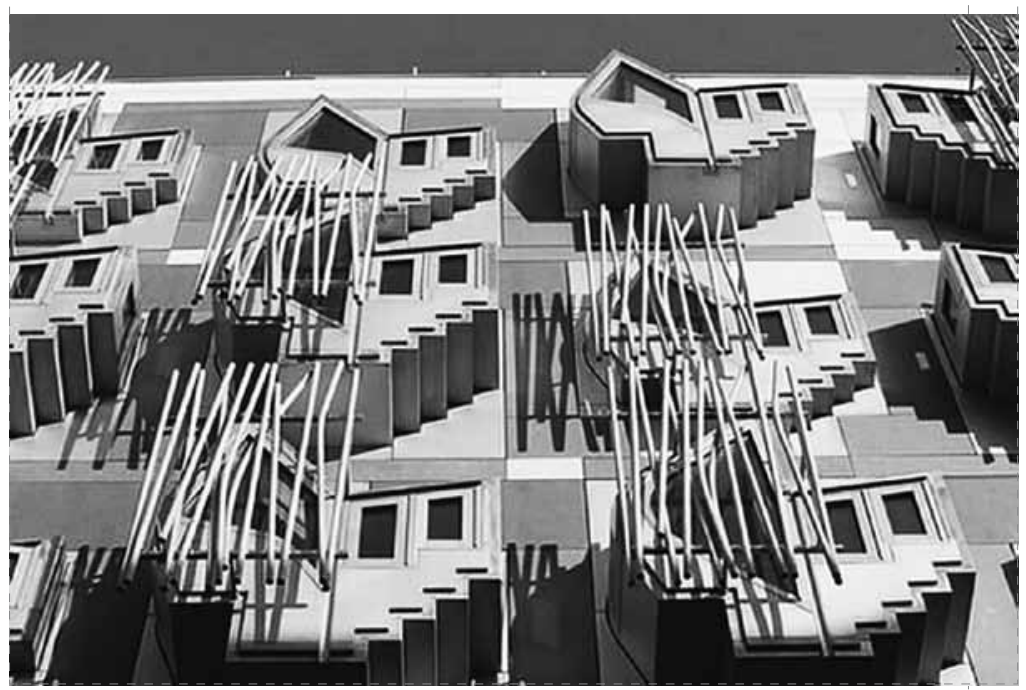

- Uno, la escala del total que determina dimensiones genéricas a los motivos principales.

- Otro, la escala de los detalles que aparece en dos niveles: el tamaño relativo de los elementos componentes y la escala de resolución que le damos a cada uno» (el grano) ${ }^{[10]}$.

Al resolver por ejemplo la subdivisión de cristales, barandas o pasamanos, la juntura de materiales, etc., estamos en la escala menor de «detalle arquitectónico». Y aún si éstos son elementos de gran tamaño, no son considerados «motivo» si no son capaces de caracterizar el objeto como un ingenio descriptivo de su arquitectura. Bajo esta distinción, el Museo Guggenheim de Frank Gehry en Bilbao, España, está conformado sólo de «motivos» que lo caracterizan con una tecnología ad-hoc donde los detalles son indiferentes. En contrario, arquitectos como Carlo Scarpa han hecho de los detalles, el «motivo descriptivo» de cada obra.

Otra distinción entre el detalle y el motivo radica en su grado de articulación dimensional según la escenicidad en que se encuentran, el ángulo de visión y punto de vista: "Cada parte del edificio debe diseñarse para la distancia a que será vista... Si un remate de edificio será siempre visto sólo desde la distancia o desde abajo, la escala del piso de remate debe crecer conforme se eleva», dice Ernst, citando el
Palacio Farnese, cuyo nivel superior es más alto para que «no disminuya» por efecto de la perspectiva en cercanía, lo cual ensayará como motivo Rafael Moneo en la actualidad.

Dado lo caracterizante de los motivos y su demanda de escenicidad, los arquitectos se ven obligados a decidir la escala a la que éstos deben percibirse (próxima, cercana o lejana) y el efecto que tendrán en el total, para que apoyen esa significación desde donde se infiere la escala del total. Saben que es la escala que asignamos al "motivo» -y no tanto la del total- la que nos comunica «el código» para entender el relato total.

Y si todo código es social por definición, en su rol comunicativo inmediato los «motivos» deben dirigirse a la idiosincrasia local. Es lo que sugiere la escala elegida para los «motivos» que proyectó el catalán Eric Miralles -y que terminara su esposa Benedetta Tagliabuhe- en el edificio del Parlamento de Escocia. Allí la escala que asumen los motivos dentro de las reducidas dimensiones del total, muestra la comprensión del autor extranjero hacia el lugar y la idiosincrasia insular de lo pequeño, confirmando otras observaciones que hiciera Ernst hace un siglo: "Cuando en el pasado un «orden» establecía la guía principal de la composición, el problema de la escala de cada motivo era simple. Pero en ausencia de un orden principal, el problema crece... mientras más simple el edificio, más grande el «motivo de escala» que requiere (...)» ${ }^{[11]}$.

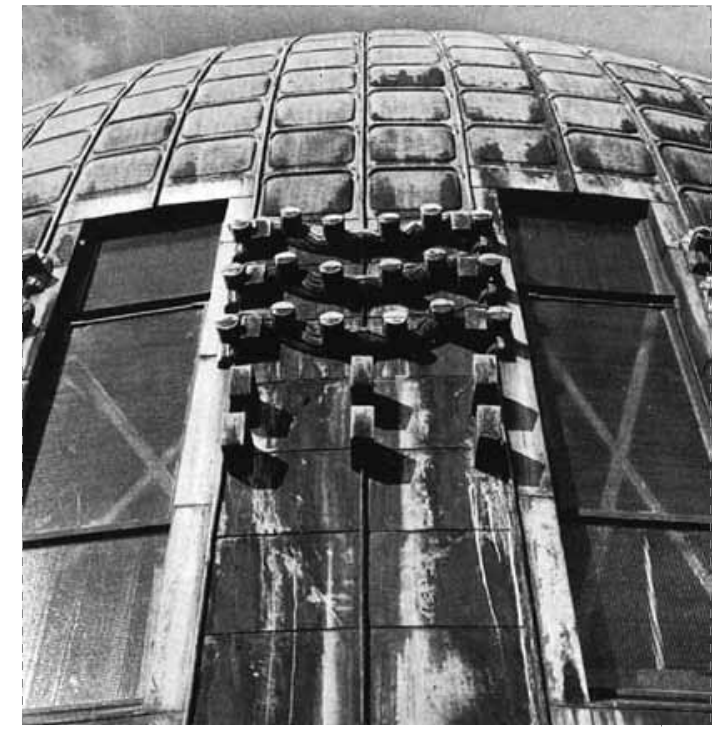

St. Leopold Am Steinhof, Viena. Otto Wagner (fuente: Otto Wagner, Ed. Gustavo Gili, Barcelona, 1984)

Entonces, si al decidir las escalas de un proyecto, la escala del «motivo» es la variable independiente -o de libre elección-, la del detalle resulta ser a menudo una variable dependiente. Ésta última, en la mayoría de los casos dependerá de la escala global que se busca relevar; a no ser que se persiga determinado efecto tectónico en el todo, dado precisamente por el grano sensual del detalle -o por su absoluta ausencia-, transformándolo en «motivo»: la cúpula de la Iglesia de St. Leopold en Viena, de Otto Wagner (1907) ya mostraba el detalle de uniones como «motivo»
[10] Ernst, Jonson. «Scale in Architecture». En: Architectural Record N 52 (LII), November, 1922, pp. 449-451. [11] Ernst, Jonson. Ibid. 


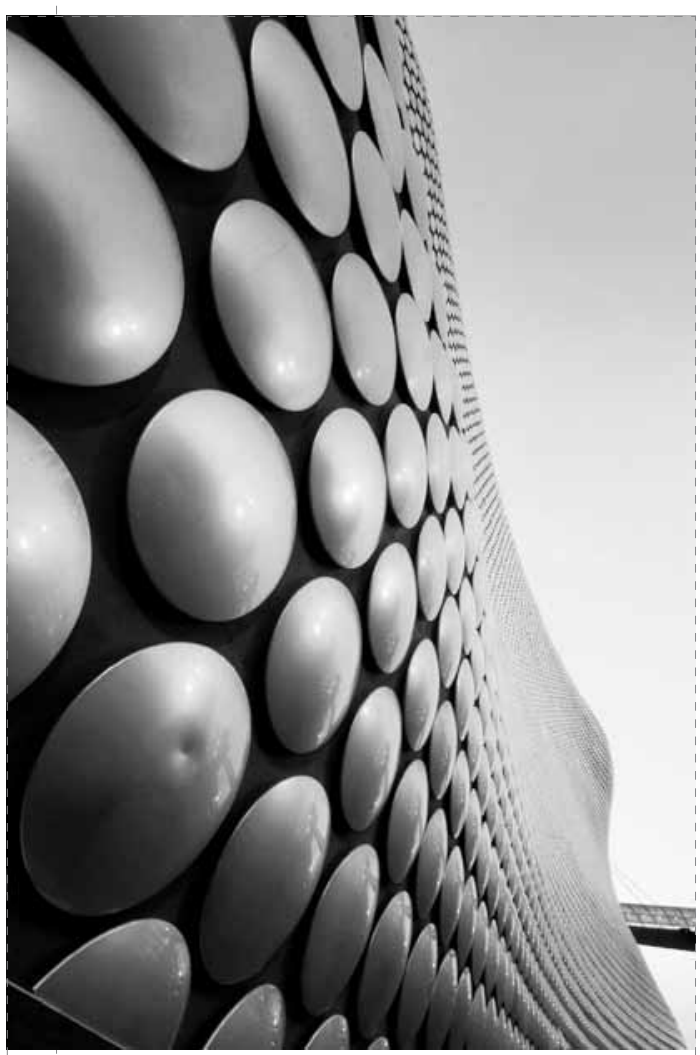

Kunsthaus Graz, Colin Fournier - Peter Cook (fotografía: Darrell Godliman en www. flickr.com) caracterizante; mientras lo contrario sucede hoy en el revestimiento de la Kunsthaus Graz, de Colin Fournier y Peter Cook, cuyo «grano» colosal, por ser homogéneo, se hace «in-significante», si bien aumenta la escala de la piel y, con ello, la del volumen. Siempre el «motivo» de ausencia de detalles o su multiplicación hasta saturar, otorga una continuidad que si bien resalta las sensualidades del manto, la obsesiva dilución de los detalles hace equívoca su escala.

Si aún en el presente la "escala de detalle» varía conforme lo hace la "escala del motivo», las gradientes y dependencias entre ambas escalas son progresivamente menos estrictas que lo que sucedía hasta muy recientemente. No obstante, vemos que aún hoy se hace de algún "detalle» un "motivo» -o que un «motivo» se vuelve un total-; lo que hace evidente que los dogmas a partir de De Stijl quedaron como anécdota, y que persiste un acertijo no resuelto en la arquitectura que mantiene latente la dialéctica entre tales categorías.

\section{Referencias bibliográficas}

Boullée, Etienne-Louis. «Arquitectura. Ensayo sobre el Arte». Ed. Gustavo Gili, Col. Punto y Línea, Barcelona, 1985

Ernst, Jonson. «Scale in Architecture». En Architectural Record N 52 (LII), noviembre, 1992

Foscari, Mario. «The «Tell-the Tail» Detail». En "Theorizing a New Agenda for Architecture. An Anthology of Architectural Theory 1965-1995». Editor. Kate Nesbitt, Princeton Architectural Press, 1998

Frampton, Kenneth. «Historia Crítica de la Arquitectura Moderna», Ed. Gustavo Gili. Barcelona, 2002

Gregotti, Vittorio. «The Excercise of Detailing». En Casabella N 492, junio 1983.
Letelier, Sofía. «Lectura e ideación de la Escala y "escalaje» en arquitectura: inteligencia visual que adquiere identidad en la geografía». Capítulo 30, s/ editar, Madrid, Noviembre. www.aq.upm.es, 2007.

Ramazziotti, Juan. «Huellas Espaciales de la dimensión humana. Hacia un Modelo Integral de las escalas dimensionales del habitar». Tesis Doctoral, ETSAM, UPM, 1998.

Van Eck, Carolina. «Architecture, Lenguage and Rethoric in Alberti»s De Re Aedificatoria» (capítulo). En «Architecture and Language. Constructing Identity in European Architecture. c.1000-1650». Klark, G / Crossley, P.(editores), Cambridge Univ. Press, London, 2000.

\section{Referencias fotográficas}

Figura 1: Libro Opere Varie di Architettura. G.B. Piranesi. 1999, Instituto Juan Herrera, pág. 8. Madrid.

Figura 2: www. frdmoptn.exblog.jp

Figura 3: Myk Reeve. Archivo de wikimedia commons. Se autoriza la copia, distribución y/o modificación de este documento bajo los términos de la Licencia de documentación libre GNU).

Figura 4: Fotografía Myrabella, Archivo de wikimedia commons. Se autoriza la copia distribución y/o modificación de este documento bajo los términos de la Licencia de documentación libre GNU).

Figura 5: Fotografía JCRA. Archivo de wikimedia commons. Se autoriza la copia, distribución y/o modificación de este documento bajo los términos de la Licencia de documentación libre GNU).

Figura 6: www.arkinetia.com

Figura 7: www.arkinetia.com

Figura 8: Libro «Otto Wagner», Ed. Gustavo Gili, Barcelona, 1984.

Figura 9: Darrell Godliman en www.flickr.com 